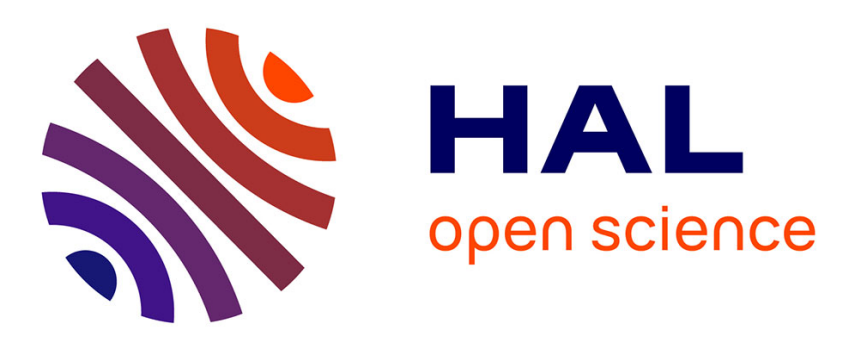

\title{
BROADENING OF ELECTRON ENERGY LEVELS AND NUCLEAR SPIN-LATTICE RELAXATION IN SMALL SUPERCONDUCTORS
}

\author{
E. Imánek
}

\section{- To cite this version:}

E. Imánek. BROADENING OF ELECTRON ENERGY LEVELS AND NUCLEAR SPIN-LATTICE RELAXATION IN SMALL SUPERCONDUCTORS. Journal de Physique Colloques, 1977, 38 (C2), pp.C2-79-C2-82. 10.1051/jphyscol:1977216 . jpa-00217055

\section{HAL Id: jpa-00217055 https://hal.science/jpa-00217055}

Submitted on 1 Jan 1977

HAL is a multi-disciplinary open access archive for the deposit and dissemination of scientific research documents, whether they are published or not. The documents may come from teaching and research institutions in France or abroad, or from public or private research centers.
L'archive ouverte pluridisciplinaire HAL, est destinée au dépôt et à la diffusion de documents scientifiques de niveau recherche, publiés ou non, émanant des établissements d'enseignement et de recherche français ou étrangers, des laboratoires publics ou privés. 


\title{
BROADENING OF ELECTRON ENERGY LEVELS AND NUCLEAR SPIN-LATTICE RELAXATION IN SMALL SUPERCONDUCTORS
}

\author{
E. ŠIMÁNEK \\ Department of Physics, University of \\ California, Riverside, California 92502, U.S.A.
}

\begin{abstract}
Résumé. - Pour expliquer l'absence d'effets de quantification des niveaux dans les petites particules nous proposons un mécanisme d'élargissement des niveaux mettant en jeu un passage des électrons d'un grain à l'autre par effet tunnel et thermiquement activé par interaction porteur-porteur. Nous étudions aussi l'élargissement dû aux fluctuations de supraconductivité. Ce dernier mécanisme n'agit qu'en présence d'un élargissement monoélectronique.
\end{abstract}

Abstract. - To explain the absence of level quantization effects in small particles we propose a level broadening mechanism involving intergrain electron tunneling, thermally activated by carrier-carrier interaction. We also investigate the quasiparticle broadening due to superconducting fluctuations. The latter mechanism is found operative only in the presence of one-electron broadening.

1. Introduction. - As pointed out by Kubo [1], the nuclear spin-lattice relaxation rate in fine metallic particles should be suppressed by the discretness of electron levels. Subsequent measurements on aluminum and copper powders made by Kobayashi et al. [2], however, did not show such a suppression. This discrepancy became more apparent after the recent study by Takashi $e t$ al. [3]. In this latter work a good agreement was obtained between the measured relaxation rates for small aluminum particles and the theory of Sone [4] which did not include any level quantization at all.

The present work is an attempt to resolve the above discrepancy. We are guided by the idea that the nuclear spin-lattice relaxation process will be enabled if the electrons of the small grain are coupled to the surrounding thermal reservoir [1]. To explain the spin-lattice relaxation rates of ref. $[2,3]$, this coupling should produce broadening of electron levels which is comparable with the electron level spacing due to finite size. Takahashi et al. [3] suggested that coupling of electrons to superconducting fluctuations may be responsible for the broadening of the discrete levels. In section 2 we analyze this possibility and come to the conclusion that superconducting fluctuations in an isolated grain do not broaden the levels in accord with the reversible behaviour of a finite close system. Section 3 is devoted to a description of a new mechanism for broadening of electron levels in fine particle powders. It is based on the observation that in actual samples the particles have a tendency to form clusters. This enables electron hopping between grains which is somewhat similar to the impurity hopping in semiconductors [5]. We also discuss the mechanisms which couple the electron to the thermal reservoir and propose one which involves the time-dependent electric field fluctuations produced by the thermal motion of the reservoir carriers. The resulting hopping rate is much stronger than that due to the phonon assisted hopping - a process well known in impurity conduction [5]. Finally, we compare this broadening mechanism with some of the present experimental data on small particle powders.

2. Interaction of electrons with fluctuating Cooper pairs. - Fluctuating Cooper pairs are described by a $t$-matrix, which in a small grain has no spatial dependence and is only a function of frequency $[6$, 7]. The role of the $t$-matrix in the mechanism of level broadening can be understood by referring to figure 1a which depicts the Dyson equation for electron propagator renormalized by pair-

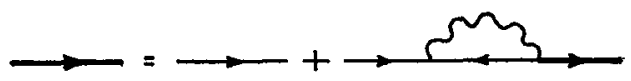

a)

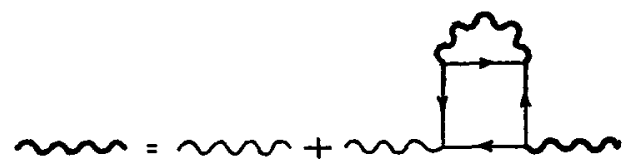

b)

FIG. 1. - a) Dyson equation for electron propagator renormalized by the interaction with pair fluctuations. Light straight lines : bare electron propagator; heavy straight lines : fluctuation renormalized electron propagator; wavy line : $t$ matrix.

b) Dyson equation for the $t$-matrix in Hartree Approximation Straight lines of the box diagram : electron propagators ; light wavy line : $t$-matrix in the RPA approximation; heavy wavy line: $t$-matrix in Hartree approximation. 
fluctuations [7]. The inverse electron lifetime will be given by the imaginary part of the self-energy. When the poles of the $t$-matrix are imaginary, corresponding to a relaxation of Cooper pairs, there will be a finite lifetime-broadening of the electron levels. This is the case considered previously by Patton [6] who starts from the RPA $t$-matrix given by

$$
t_{\mathrm{RPA}}^{-1}(\omega)=N_{0} \Omega\left[1 \mathrm{n}\left(T / T_{\mathrm{c}}\right)-i \omega \pi / 8\right]
$$

where $N_{0}$ is the electron density of states at the Fermi level and $\Omega$ is the volume of the particle. We note that the imaginary pole in eq. (1) is a direct consequence of the use of free-electron propagators with continuous energy spectrum [6]. Therefore it is of interest to calculate the $t$-matrix in the discrete electron level scheme of a small particle. Following Kadanoff and Martin [8] we have in this case

$$
\begin{aligned}
& {\left[t_{\mathrm{RPA}}^{\mathrm{s})}(\omega)\right]^{-1}=\frac{1}{V}+\sum_{k} \frac{1}{\omega-2 \varepsilon_{k}} } \tanh \frac{\varepsilon_{k}}{2 T}= \\
&=\frac{1}{V}+F(\omega, T)
\end{aligned}
$$

where $V$ is the strength of the electron-electron interaction and $\varepsilon_{k}$ are the discrete energy levels. The poles of $t \mathrm{KP}_{\mathrm{A}}(\omega)$ are obtained graphically by plotting the function $F(\omega, t)$, and looking for intersections of the latter with the $-\frac{1}{V}$ line. (See Fig. 2). For $T>T_{\mathrm{c}},|F(0, T)|<1 / V$ and intersections at finite $\omega$ appear, corresponding to real poles. For $T<T_{\mathrm{c}},|F(0, T)|>1 / V$, and became imaginary indicating the onset of instability with respect to the formation of Cooper pairs [8]. In a small supercon-

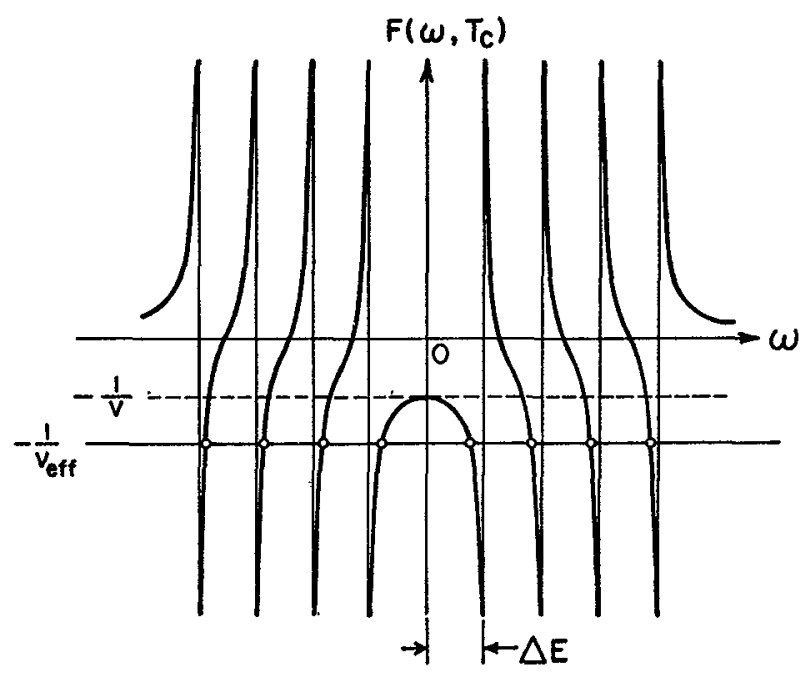

FIG. 2. - A plot of the function

$$
F\left(\omega, T_{c}\right)=-\sum_{k} \frac{1}{2 \varepsilon_{k}} \tanh \frac{\varepsilon_{k}}{2 T_{c}} .
$$

Intersections of $F\left(\omega, T_{\mathrm{c}}\right)$ with the line $-\frac{1}{V_{\text {eff }}}$ determine the poles of the function $t_{\mathrm{H}}^{(s)}(\omega)$ at $T=T_{\mathrm{c}}$. The function $F\left(\omega, T_{c}\right)$ is shown to touch the $-\frac{1}{V}$ (dashed line at $\omega=0$, in accord with the RPA definition of $T_{\mathrm{c}}$. The energy levels $\varepsilon_{k}$ are separated by a constant difference $\Delta E$. ductor, the inclusion of the interactions between fluctuations is known to smear the phase transition $[6,7]$. In the following we show, that, if these interactions are taken into account in a self-consistent Hartree approximation, the resulting $t$-matrix $t(\omega)$ exhibits only real roots.

From the Dyson equation shown in figure $1 \mathrm{~b}$ we obtain

$\left[t_{\mathrm{H}}^{(\mathrm{s})}(\omega)\right]^{-1}=\frac{1}{V}+g(T) t_{\mathrm{H}}^{(\mathrm{s})}(\omega=0)+F(\omega, T)$.

The parameter $g(T)$ is obtained, by calculation of the box diagram in figure $1 \mathrm{~b}$, in the form

$$
g(T)=T^{2} \sum_{k} \sum_{m} \frac{1}{\left(\omega_{m}^{2}+\varepsilon_{k}^{2}\right)^{2}}
$$

where $\omega_{m}=\pi(2 m+1) T$. Solving eq. (3) for $t^{(s)}(\omega=0)$ we get

$$
\begin{aligned}
t_{\mathrm{H}}^{(\mathrm{s})}(\omega=0)= & -\frac{1}{2 g(T)}\left[\frac{1}{V}+F(0, T) \mp\right. \\
& \mp \sqrt{\left.\left[\frac{1}{V}+F(0, T)\right]^{2}+4 g(T)\right]} .
\end{aligned}
$$

The proper branch of this solution is the one which involves the upper sign on the r.h.s. of eq. (5). In fact, if one takes the limit $g \rightarrow 0$ of the latter one recovers the RPA result $t_{\mathrm{RPA}}^{(\mathrm{s})}(\omega=0)$. From eqs. (4) and (5) it follows that $t_{\mathrm{H}}^{(\mathrm{s})}(\omega=0)>0$. Eq. (3) then implies

$$
\frac{1}{V_{\text {eff }}}=\frac{1}{V}+g(T) t_{\mathrm{H}}^{(\mathrm{s})}(\omega=0)>|F(0, T)|
$$

Consequently there are intersections of the $F(\omega, T)$ curves and the $-\frac{1}{V_{\text {eff }}}$ line, corresponding to real poles of $t$ (S) $(\omega)$.

This result in in accord with the above assertion that there should be no superconducting instability in a small superconductor. Furthermore, if the self-energy in figure 1 is calculated with the use of $t_{\mathrm{H}}^{(\mathrm{s})}(\omega)$ there will be no lifetime broadening due to superconducting fluctuations; since the self-energy has no imaginary part. Hence, we conclude that in an isolated grain, the renormalization by fluctuations in not a sufficient mechanism for lifetime broadening of the discrete electron levels.

However, if the electrons of the grain are coupled by some other mechanism to the thermal reservoir we have an open system with irreversible behaviour [9]. This can be seen by inserting complex energies $\varepsilon_{k}$ into (3), or simply by working with continous energy spectrum appropriate for electrons in an infinite system [6]. The resulting $t$-matrix has then complex roots like in eq. (1) yielding a broadening [6]

$$
\delta E \simeq \frac{T_{c}}{3\left(\Omega N_{0} T_{c}\right) \varepsilon^{2}}=\frac{\Delta E}{3 \varepsilon^{2}}
$$


where $\varepsilon=\left(T-T_{\mathrm{c}}\right) / T_{\mathrm{c}}$ and $\Delta E$ is the level separation due to finite size of the grain [1]. This result indicates that the fluctuation mechanism [3] is quite effective for $T \simeq T_{c}$, provided there is a coupling of electrons to the thermal reservoir.

3. Thermally activated intergrain hopping. - We describe now a mechanism for the above coupling which should be operative in samples where the fine particles are in a good mechanical contact with each other [10]. Electron micrographs of the powder samples show frequently a tendency of the particles to form clusters. This can be understood by taking into account the attraction between particles due to the Van der Waals force. For an orientational estimate of the latter we calculate the energy of attraction $U$ of two metallic spheres of radius $R=100 \AA$, separated by a distance of closest points $s=5 \AA$. Following ref. [11], we have

$$
U \simeq-\frac{R}{8 \pi s} \hbar \omega \simeq 10^{5} k_{\mathrm{B}}
$$

where $k_{\mathrm{B}}$ is the Boltzmann constant and $\hbar \omega$ is the plasmon energy, which, for metals, is of the order of $10 \mathrm{eV}$. Hence it is practically impossible to dissociate the particle-pair at room temperatures.

As a result of this cluster formation, most of the particles in the sample experience strong perturbations due to thermal excitations of the neighbouring grains. These perturbations will produce transitions between electron energy levels. We consider transitions which involve thermally activated hopping of electrons between grains [10]. The latter mechanism is known to be responsible also for the electrical conduction in granular films in the dielectric regime [12]. We note that the proposed intergrain hopping does not necessarily imply a high electrical conductivity of the powder as a whole. Imperfect packing will inhibit the percolation of electrons across the sample. In addition, the fluctuations of the size of the particles produce energy level mismatch between neighboring grains thus contributing to an Anderson-type of localization of the electrons [13].

To calculate the hopping rate between the neighbouring grains $a$ and $b$ we first consider the quantum mechanical mixing of the corresponding electron orbitals $\phi_{\mathrm{a}}$ and $\phi_{\mathrm{b}}$ yielding orbitals of the pair-complex [5]

$$
\left.\begin{array}{l}
\phi_{\mathrm{i}}=\phi_{\mathrm{a}}+\gamma \phi_{\mathrm{b}} \\
\phi_{\mathrm{b}}=\phi_{\mathrm{b}}-\gamma \phi_{\mathrm{a}}
\end{array}\right\}
$$

where $\gamma \simeq T^{1 / 2}, T$ being the transmission coefficient of the potential barrier due to oxide coating of the particles. The electron is initially in orbital $\phi_{i}$ with energy $E_{\mathrm{i}}<E_{\mathrm{j}}$ (we assume $E_{\mathrm{a}}<E_{\mathrm{b}}$ ). A real transition to orbital $\phi_{j}$ takes place provided the energy difference $E_{\mathrm{j}}-E_{\mathrm{i}}$ is supplied by the thermal reservoir. A conventional mechanism for the electron-reservoir interaction is the electron-phonon coupling familiar from impurity conduction in semiconductors [5]. For small particles the relevant deformation potential arises from vibrationally induced changes of their volume. Numerical estimates of the hopping rate due to the latter mechanism were made [14]. For aluminum particles of radius $100 \AA$ and at $T \simeq 1 \mathrm{~K}$ we find a rate which is one to two orders of magnitude too small to account for the required width of levels.

A more effective electron-reservoir coupling results from interaction between the electron and the electrostatic potential fluctuations $V(r, t)$ due to the thermal motion of the carriers surrounding the grain a. The corresponding interaction Hamiltonian is

$$
\begin{aligned}
& \mathscr{H}=-e \int \psi^{+} \psi V(r, t) \mathrm{d}^{3} r= \\
& =e \gamma c_{\mathrm{j}}^{+} c_{\mathrm{i}}\left(V_{\mathrm{a}}-V_{\mathrm{b}}\right)+\text { h.c. }
\end{aligned}
$$

where $\psi=c_{\mathrm{i}} \phi_{\mathrm{i}}+c_{\mathrm{j}} \phi_{\mathrm{j}}$. The operator $c_{\mathrm{j}}^{+} c_{\mathrm{i}}$ acts to transfer the electron from $\phi_{\mathrm{i}}$ to $\phi_{\mathrm{j}}$. The electrostatic potential difference between the grains $\Delta V(t)=V_{\mathrm{a}}-V_{\mathrm{b}}$ is a random function of time. The fluctuations of $\Delta V(t)$ induce energy conserving transitions $i \rightarrow j$ with the probability rate

$W_{\mathrm{ij}}=e^{2} \gamma^{2} \int_{\infty}^{\infty} \mathrm{d} t \mathrm{e}^{i\left(\omega_{1}-\omega_{\mathrm{i}}{ }^{2} t\right.}\langle\Delta V(t) \Delta V(0)\rangle$.

The contribution to $\Delta V$ due to distant carriers is strongly diminished by the presence of DebyeHückel screening [15] and one can calculate $\langle\Delta V(t) \Delta V(0)\rangle$ by including only the carriers hopping on and off the nearest-neighbour grains [10]. Denoting the average time of residence of a carrier on a given grain by $\tau$ we then have from (11)

$$
W_{\mathrm{ij}}=\frac{e^{2} \gamma^{2} \tau}{1+\left(\omega_{\mathrm{i}}-\omega_{\mathrm{j}}\right)^{2} \tau^{2}} \exp \left(-E_{\mathrm{c}} / 2 T\right) \sum_{r=1}^{z}\left(\Delta V^{(r)}\right)^{2}
$$

Where $\Delta V^{(r)}$ is the potential due to $r$-th neighbours, $z$ is the coordination number of grain a, and $E_{\mathrm{c}}$ is the charging energy [12]. We note that the factor $\exp \left(-E_{\mathrm{c}} / 2 T\right)$ gives the probability of a grain to be singly ionized. To estimate the lifetime broadening $\delta E=\hbar / \tau$, we assume an octahedral coordination and calculate $1 / \tau$ from (12) self-consistently, with use of relation $1 / \tau=6 W_{i j}$. For $\left(\omega_{i}-\omega_{j}\right)^{2} \tau^{2} \ll 1$, we get

$$
\delta E=0.1 \gamma \exp \left(-E_{\mathrm{c}} / 4 T\right)\left(e^{2} / R\right) .
$$

The charging energy $E_{c}$ can be estimated using the model of a concentric capacitor [12]. For particles of radius $R=100 \AA$ separated by an oxide layer of dielectric constant $\varepsilon=5$ and of thickness $s=5 \AA$ we obtain $E_{\mathrm{c}} \simeq e^{2} s / \varepsilon R^{2} \simeq 1.4 \times 10^{-3} \mathrm{eV}$. Inserting this value into (13) we get, at $T \simeq 1 \mathrm{~K}$, a complete smearing of levels $\left(\delta E \simeq \Delta E=10^{-5} \mathrm{eV}\right)$ for $\gamma=5 \times 10^{-2}$. This is a $10^{-2}$ smaller value than that required by the phonon assisted hopping [14] and 
leads to an acceptable barrier transmission coefficient [12].

The $R^{-2}$ dependence of $E_{\mathrm{i}}$ implies, via eq. (13), a pronounced dependence of $\delta E$ on the particle size. Recent far-infrared studies [16] show a distinct energy level structure for smaller particles ( $2 R=150 \AA$ ) and a smeared spectrum for larger particles ( $2 R=400 \AA$ ), which is in accord with eq. (13) (see the note added in proofs). Since the intergrain hopping makes the electron propagator similar to that of a dirty superconductor [17], one can understand why the theory of Sone [4], based on such propagators, gives a good agreement with experiment [3].

I should like to thank Professor D. MacLaughlin for many helpful discussions and critical reading of the manuscript.

Note added in proofs : A more recent study by Granqvist et al. (Phys. Rev. Lett. 37 (1976) 625) did not reveal this level structure for ultrafine Al particles $(2 R=50 \AA)$. The far-infrared absorption is probably dominated by the dielectric losses in the amorphous surface oxide layer (see E. Šimánek, Phys. Rev. Lett. 38 (1977) 1161).

\section{References}

[1] Kubo, R., J. Phys. Soc. Japan 17 (1962) 975.

[2] Kobayashi, S., Takahashi, T., and Sasaki, W., J. Phys. Soc. Japan 31 (1971) 1442.

[3] Takahashi, T., Kobayashi, S., and Sasaki, W., Solid State Commun. 11 (1975) 681.

[4] Sone, J., J. Low Temp. Phys. 23 (1976) 699.

[5] Mort, N. F., and Davis, E. A., in Electronic Processes in Non-crystalline Materials (London: Clarendon Press) 1971 , p. 104

[6] Patton, B., Phys. Rev. Lett. 27 (1971) 1273.

[7] ŠIMÁNEK, E., in Proceedings of the International School of Physics Enrico Fermi, on Local Properties at Phase Transitions, Course LIX, July 1973 (Academic Press : New York and London) 1975 , p. 838.

[8] Kadanoff, L. P., and Martin, P. C., Phys. Rev. 124 (1961) 6.
[9] DAvies, P. C.W., in The Physics of Time Asymetry (University of California Press : Berkeley and Los Angeles) 1974, p. 154.

[10] Š́mÁnek, E., Phys. Lett. 59A (1976) 231.

[11] Mitchell, D. J., and Ninham, B. W., J. Chem. Phys. 56 (1972) 1117

[12] Abeles, B., Sheng, P., Coutts, M. D., and Arie, Y., Adv. Phys. 24 (1974) 407

[13] Anderson, P. W., Phys. Rev. 109 (1958) 1492.

[14] ŠIMÁNEK, unpublished results.

[15] Debye, P., and Huckel, W., Phys. Z. 24 (1923), 185, 305.

[16] Tanner, B. D., Sievers, A. J., and Buhrman, R. A., Phys. Rev. B 11 (1975) 1330.

[17] Abeles, B., Cohen, R. W., and Stowell, W. R., Phys. Rev. Lett. 18 (1967) 902. 